Mathematical Problems of Computer Science 53, 7-13, 2020.

UDC 510.6

\title{
Polynomial Bounded Proof Complexities for Some Classes of DNF-Tautologies
}

\author{
Garik V. Petrosyan \\ Yerevan State University \\ e-mail: garik.petrosyan.1@gmail.com
}

\begin{abstract}
In this paper, we present the results on Frege proof complexities of some DNFtautologies. At first we introduce the notion of complete DNFs and prove that complete DNFs are tautologies, we also show that if the proof complexities for the set of complete DNFs are polynomially bounded, then the set of DNF-tautologies $D$, for which the number of non-negated variables in every conjunct is $O(\log (D))$, also has polynomially bounded proof complexities. Later we show that the set of all balanced DNF-tautologies has polynomial proof complexities.
\end{abstract}

Keywords: Frege systems, Proof complexity, DNF, complete DNF, balanced formulas.

\section{Introduction}

One of the most fundamental problems of the proof complexity theory is to find an efficient proof system for classical propositional calculus. There is a widespread understanding that polynomial-time computability is the correct mathematical model of feasible computation. According to the opinion, a truly "effective" system should have a polynomial-size $p(n)$ proof for every tautology of size n. In [1], Cook and Reckhow named such a system a super system. They showed that $N P=c o N P$ iff there exists a super system. It is well known that many systems are not super. This question about the Frege system, the most natural calculi for propositional logic, is still open.

In many papers, some specific sets of tautologies are introduced, and it is shown that the question about polynomially bounded sizes for Frege-proofs of all tautologies is reduced to an analogous question for a set of specific tautologies. In particular, Lutz Strasburger introduced in [2] the notion of balanced formulas and showed that if there are polynomially bounded Frege proofs for the set of balanced tautologies, then the Frege systems are super. An analogous result for some other class of tautologies is proved in [3].

One of the well-known classes of tautologies is the class of tautologies, given in disjunctive normal form (DNF-tautologies), and it is an open question if the Frege-proof complexities for the set of DNF-tautologies have polynomial upper bounds. The Frege-proof complexities of some DNF-tautology classes are investigated in this paper. At first the notion of complete 
DNF is introduced, and it is proved that if the proof complexities for the set of complete DNFs are polynomially bounded, then the set of DNF-tautologies $D$, for which the number of non-negated variables in every conjunct is $O(\log (D))$, also has polynomially bounded proof complexities. Then it is proved that the proof complexities of the set of balanced DNF-tautologies has polynomially bounded proof complexities as well.

\section{Main Notions and Notations}

Here we give basic definitions, which are necessary to give main results.

Definition 1: A Frege system $F$ uses a denumerable set of propositional variables, a finite complete set of propositional connectives; $F$ has a finite set of inference rules defined by a figure of the form $\frac{A_{1} A_{2} \ldots A_{n}}{B}$ (the rules of inference with zero hypotheses are axiom schemes); $F$ must be sound and complete, i.e., for each rule of inference $\frac{A_{1} A_{2} \ldots A_{n}}{B}$ every truth-value assignment, satisfying $A_{1} A_{2} \ldots A_{n}$, also satisfies $B$, and $F$ must prove every tautology.

The particular choice of a language for the presented propositional formulas is immaterial in this consideration. However, because of some technical reasons, we assume that the language contains the propositional variables $p_{i}(i \geq 1)$ or $p_{i j}(i \geq 1 ; j \geq 1)$ logical connectives $\neg, \wedge, \vee, \supset$ and parentheses $($,$) . Note that some parentheses and \wedge$ can be omitted in generally accepted cases. Note that our convention for serial disjunction $A_{1} \vee A_{2} \vee \ldots \vee A_{n}$ (conjunction $\left.A_{1} \wedge A_{2} \wedge \ldots \wedge A_{n}\right)$ associated from left to right.

By $|\varphi|$ we denote the size of the formula $\varphi$ defined as the number of all entries of logical signs in it. It is obvious that the full size of the formula, which is understood to be the number of all symbols, is bounded by some linear function in $|\varphi|$.

In the theory of proof complexity, the four main characteristics of the proof are: $t$ complexity (length), defined as the number of proof steps, $l$-complexity (size), defined as the sum of sizes for all formulas in the proof (size), s-complexity (space), informally defined as the maximum of minimal sum of sizes for formulas on blackboard needed to verify all steps in the proof (formal definitions are, for example, in [2]) and w-complexity (width), defined as the maximum of sizes of the proof formulas.

Definition 2: Let $\phi$ be a proof system and $\varphi$ be a tautology. We denote by $t_{\varphi}^{\phi}\left(l_{\varphi}^{\phi}, s_{\varphi}^{\phi}, w_{\varphi}^{\phi}\right)$ the minimal possible value of t-complexity (l-complexity, s-complexity, w-complexity) for all -proofs of tautology $\varphi$. By analogy, we can define the mentioned proof complexity characteristics for the proof of any formula $A$ from premises $\Gamma$ and denote them respectively by $t_{\Gamma \vdash A}^{\phi}\left(l_{\Gamma \vdash A}^{\phi}, s_{\Gamma \vdash A}^{\phi}, w_{\Gamma \vdash A}^{\phi}\right)$.

Let $M$ be some set of tautologies.

Definition 3: We call the $\phi$-proofs of tautologies from the set $M$ t-polynomially (lpolynomially, s-polynomially, w-polynomially) bounded if there is a polynomial $p()$ such that $t_{\varphi}^{\phi} \leq p(|\varphi|)\left(l_{\varphi}^{\phi} \leq p(|\varphi|), s_{\varphi}^{\phi} \leq p(|\varphi|), w_{\varphi}^{\phi} \leq p(|\varphi|)\right)$ for all tautologies $\varphi$ from $M$.

Note that if $\phi$-proofs of tautologies from the set $M$ are $l$-polynomially bounded they are also $t$-polynomially, $s$-polynomially, $w$-polynomially bounded.

Following the usual terminology, we call the variables and negated variables literals for classical logic. The conjunct $K$ can be represented simply as a set of literals (no conjunct contains a variable and its negation simultaneously).

In [4], the notion of balanced formulas is introduced in the following way. 
Definition 4: A propositional formula is called balanced if every variable has only two occurrences in it, one positive and one with negative.

In [4], it is shown that the problem on l-polynomially bounded sizes of proofs for all tautologies is reduced to the problem on $l$-polynomially bounded sizes of proofs for all balanced tautologies.

\section{Main Results}

In this part, Frege-proof complexities for some classes of DNF-tautologies are investigated. A. Here the notion of complete DNF-tautologies is introduced, and it is proved that if the set of complete DNF-tautologies has $l$-polynomially therefore also $t$-polynomially, $s$ polynomially, $w$-polynomially bounded proofs, then the set of DNF-tautologies $D$, where the number of non-negated variable in each conjunct is $O(\log (|D|))$, also has $l$-polynomially therefore also $t$-polynomially, $s$-polynomially, $w$-polynomially bounded proofs.

Let all variables of a DNF $N_{1} \vee N_{2} \vee \ldots \vee N_{n}$ be negated variables. Conjunct $K$ is called representative for $D$ if $K$ contains at least one variable (without negation) from each $N_{i}$ $(1 \leq i \leq n)$, and every variable of $K$ is from $D$.

Definition 5: DNF $D_{1}=C_{1} \vee C_{2} \vee \ldots \vee C_{m}$ is called a completion of DNF $D_{2}=N_{1} \vee$ $N_{2} \vee \ldots \vee N_{n}$ iff for every representative $K$ of $D_{2}$ there is a $N_{i}(1 \leq i \leq n)$, which is a subset of $K$, and the expression $D=\left(N_{1} \vee N_{2} \vee \ldots \vee N_{n}\right) \vee\left(C_{1} \vee C_{2} \vee \ldots \vee C_{m}\right)$ is called a complete DNF.

Theorem 1: Complete DNFs are tautologies.

Proof. Let's assume the opposite: there is a complete DNF $D=\left(N_{1} \vee N_{2} \vee \ldots \vee N_{n}\right) \vee\left(C_{1} \vee\right.$ $\left.C_{2} \vee \ldots \vee C_{m}\right)$ such that it is not a tautology. If $D=0$ in any collection, then $N_{1} \vee N_{2} \vee \ldots \vee N_{n}$ should also be 0 in that collection. $N_{1} \vee N_{2} \vee \ldots \vee N_{n}=0$, only if each conjunct is equal to 0 , therefore, we have that in each conjunct $N_{i}(1 \leq i \leq n)$ there is a literal equal to 0 . If we consider the conjunct $P$ constructed by the conjunction of the variables of these literals, as we have a complete DNF, there is a $C_{j}(1 \leq j \leq m)$ such that $C_{j}$ is a subset of $P$, hence $C_{j}=1$, but we have assumed that $D=0$, which is a contradiction.

From this proof it is easy to see that all DNF-tautologies, the conjuncts of which do not contain a variable and a negated variable simultaneously, are complete DNFs. To evaluate the proof complexities of DNF-tautologies by reducing the proof to the proof of complete DNFtautologies, we must use some transformations of formulas, therefore we give the following auxiliary statements, which are used to perform those transformations.

Lemma 1: For all formulas $A, B$ and $C$, the set of the following formulas

1. $A \vee B \supset C \equiv(A \supset C) \wedge(B \supset C)$

2. $A \supset A \vee B$

3. $A \supset(B \vee C) \equiv(A \supset B) \vee(A \supset C)$

4. $B \supset(A \supset A \wedge B)$

5. $(A \supset B) \supset((A \vee C) \supset(B \vee C)$

has l-polynomially bounded proofs. 
Proof. The proof is obvious.

Most DNF-tautologies, the proof complexities of which are being investigated, have small conjuncts sizes compered to their size. Using complete DNFs, we can construct polynomial proofs for such DNF-tautologies. The following theorem gives such a construction.

Theorem 2: If the set of all complete DNFs has l-polynomially bounded proofs then, the set of DNF-tautologies D, where the number of non-negated variables in each conjunct is $O(\log (|D|))$, also has l-polynomially bounded proofs.

Proof. Let's consider $D$ DNF-tautology, where the number of non-negated variables in each conjunct is $O(\log (|D|))$. For each conjunct, we separate the sub-conjunct of variables with positive entrance and the set of all such conjuncts and its subsets we denote by $P$. Since the number of non-negated variables in each conjunct is $O(\log (|D|))$, there is such a polynomial $p()$ function that $|P| \leq p(|D|)$.

If $\mathrm{DNF}$ is a tautology, it should have at least one conjunct, where all the variables are negated, otherwise it does not cover the 0 point we denote the disjunction of all such conjuncts by $N$. If $D$ is a tautology, then there is a completion of $N$ such that all conjuncts are from $P$. If such a completion did not exist, we would take a conjunct constructed by taking one variable from each conjunct of $N$, and this conjunct would not be covered by $P$, therefore if we set the values of these variables 1 and the values of all other variables 1 , the value of $D$ will be 0 . Let's denote that completion by $C . N \vee C$ is a completion DNF; to prove $D$, we need to prove $N \vee C$ and $N \vee C \supset D$, the first part follows from our condition.

Let's prove the second part $N \supset D$. From Lemma 1.1 we get two tautologies to prove $N \supset D$ and $C \supset D$. From Lemma 1.2 we get the polynomial proof of $N \supset D$. Now let's prove $C \supset D$. Suppose $C=C_{1} \vee C_{2} \vee \ldots \vee C_{m}$, where $C_{i}(1 \leq i \leq m)$ is a conjunct. Using Lemma 1.1, we can convert $C \supset D$ into $\left(C_{1} \supset D\right) \wedge\left(C_{2} \supset D\right) \wedge \ldots \wedge\left(C_{m} \supset D\right)$. As $C_{i}(1 \leq i \leq m)$ is a conjunct, we can prove each one alone and later join them with $\wedge$. Note that the number of such $C_{i}$ conjuncts is less than $P$, therefore it has a polynomial upper bound. Using Lemma 1.4 and 5, we can reduce the proof of each $\left(C_{i} \supset D\right)(1 \leq i \leq m)$ to a new DNF, which also satisfies the condition that the number of non-negated variables in each conjunct is $O(\log (|D|))$. We can use the same technique to prove these new tautologies and note that their $P$ sets are subsets of our initial $P$ set, therefore we will use only the first $P$. If we continue the proof in this way, we will have several tautologies, the number of which is polynomial from $|D|$, and each reduction is performed using polynomial steps and polynomial formulas, therefore the proof is $l$-polynomially bounded.

Corollary 3: If the set of complete DNFs has l-polynomially bounded proofs, than the set of $D N F$-tautologies $D$, where the number of negated variables in each conjunct is $O(\log (|D|))$, also has l-polynomially bounded proofs.

Proof. The proof can be obtained from the proof of Theorem 2 with slight changes.

B. Here, the proof complexities of some subset of balanced DNF-tautologies are investigated.

Definition 6: A DNF-tautology is correct if DNF, obtained from it by removing any conjunct, is no longer a tautology.

Lemma 2: The number of conjuncts in a balanced correct DNF-tautology with $n$ variables is $n+1$. 
Proof. We prove the lemma by induction on number $\mathrm{n}$ of variables in a balanced DNFtautology $D$. If $n=1$, we have $D=\neg p \vee p$. Suppose the statement is valid for a balanced DNF-tautology, which has $\leq n$ variables. If the number of variables is $n+1$, then the correctly balanced DNF should have at least one conjunct with at least two literals $p_{i_{1}}^{\alpha_{1}} p_{i_{2}}^{\alpha_{2}}$. After assigning $\alpha_{1}$ to the variable $p_{i_{1}}$ everywhere in the given DNF, both the number of variables and the number of conjuncts decrease by one, hence the number of conjuncts in the primary DNF should be $n+2$.

Corollary 4: Every balanced correct DNF-tautology has at least one conjunct, consisting of one literal.

Theorem 5: All balanced correct DNF-tautologies have l-polynomially bounded proofs.

Proof. Let us have a balanced correct DNF-tautology $D=D_{1} \vee D_{2} \vee \ldots \vee D_{n+1}$, which depends on the variables $p_{1}, p_{2}, \ldots, p_{n}$. We take $\neg D$ and prove the a contradiction. $\neg D=$ $N_{1} \wedge N_{2} \wedge \ldots \wedge N_{n+1}$, where $N_{i}=\neg D_{i}(1 \leq i \leq n+1)$ and $N_{i}=p_{i_{1}}^{\alpha_{1}} \vee p_{i_{2}}^{\alpha_{2}} \vee \ldots \vee p_{i_{r}}^{\alpha_{r}}$, where $\alpha_{i} \in\{0,1\}(1 \leq i \leq n+1)$. Based on $N_{i}$, we construct the formula $E_{i}$ by adding instead of every variable $p_{j}$ from $p_{1}, p_{2}, \ldots, p_{n}$ which has no occurrence in $N_{i}$, the formula $p_{1} \wedge \neg p_{1}$ on the j-th place with a disjunction. It is obvious that $N_{i}=E_{i}$, and this equivalence can be derived with polynomially bounded characteristics of proof complexities. By this notation we have that formula $\neg D$ is equivalent to formula $D^{\prime}=E_{1} \wedge E_{2} \ldots \wedge E_{n+1}$. Now we introduce new propositional variables $p_{i j}(1 \leq i \leq n+1 ; 1 \leq j \leq n)$, where $p_{i j}$ is true if the variable $p_{j}$ occurs in $N_{i}$ and is false for the opposite case, and construct on the base of $E_{i}$ new disjunctions $D_{i}^{\prime}$ by replacing both the primary literals and the formulas $p_{1} \wedge \neg p_{1}$ with the corresponding variables $p_{i j}$.

Now we consider the well-known formulas of Pigeon Hole Principle

$$
P H P_{n}=\wedge_{i=0}^{n} \vee_{k=0}^{n-1} p_{i k} \supset \vee_{k=0}^{n-1} \vee_{0 \leq i<j \leq n}\left(p_{i k} \wedge p_{j k}\right) \text {. }
$$

These formulas have $l$-polynomially bounded proofs. Using this fact, we can derive the formulas

$$
\wedge_{i=1}^{n+1} D_{i}^{\prime} \supset \vee_{j=1}^{n} \vee_{1 \leq i<k \leq n+1}\left(p_{i j} \wedge p_{k j}\right),
$$

and then we derive the formulas

$$
\vee_{j=1}^{n} \vee_{1 \leq i<k \leq n+1}\left(p_{i j} \wedge p_{k j}\right)
$$

Denote by $H_{n}$ the formulas $C_{n}=\wedge_{j=1}^{n} \wedge_{1 \leq i<k \leq n+1} \neg\left(p_{i j} \wedge p_{k j}\right)$. It is not difficult to derive formulas $H_{n} \supset C_{n}$ with a polynomially bounded proof. If for every $i, j$ we denote by $\left[p_{i j}\right]$ either the primary literal or the formula $p_{1} \wedge \neg p_{1}$ from the formula $E_{i}$, then it is obvious, that

1. $\left[H_{n}\right]=\wedge_{j=1}^{n} \wedge_{1 \leq i<k \leq n+1} \neg\left(\left[p_{i j}\right] \wedge\left[p_{k j}\right]\right)$

2. the formulas $\left[H_{n}\right],\left[H_{n}\right] \supset \vee_{j=1}^{n} \vee_{1 \leq i<k \leq n+1}\left(p_{i j} \wedge p_{k j}\right)$ and $P H P_{n}$ [5] have $l$-polynomially bounded proofs.

We have derived a contradiction and the proof is $l$-polynomially bounded. 


\section{Conclusion}

Even though the question of polynomial proofs of DNF-tautologies is open here, we gave some subsystems and showed that if complete DNF-tautologies have polynomial proof complexities, then there is a broad range of DNF-tautologies, which also have polynomial proof complexities. Balanced tautologies are as hard to prove as all tautologies; in this work we gave the polynomial proof for balanced DNF-tautologies.

\section{Acknowledgement}

This work was supported by the RA MES State Committee of Science, in the frames of the research project 18T-1B034. I am grateful to my supervisor, professor of YSU Anahit Chubaryan for her encouragement and fruitful discussions, and also for helpful suggestions, which improved and expanded the results.

\section{References}

[1] S. A. Cook and A. R. Reckhow, "The relative efficiency of propositional proof systems", Journal of Symbolic logic, vol. 44, pp. 36-50, 1979,

[2] N. Jakob, "Narrow proofs may be spacious: Separating space and width in resolution", SIAM Journal on Computing, vol. 39, no. 1, pp. 59-121, 2009.

[3] A. Chubaryan and G. Petrosyan, "On some properties of several proof systems for 2valued and 3-valued propositional logic", Fundamentalis Scientiam, vol. 8, no. 8, pp. 70-73, 2017.

[4] L. Strasburger, "Extension without Cut", INRIA SaclayIle-de-France and Ecole Polytechnique, LIX, Rue de Saclay, 91128 Palaiseau Cedex, France

[5] S. R. Buss, "Polynomial size proofs of the propositional pigeonhole principle", Journal of Symbolic Logic, vol. 52, pp. 916-927, 1987.

Submitted 22.01.2020, accepted 12.05.2020. 


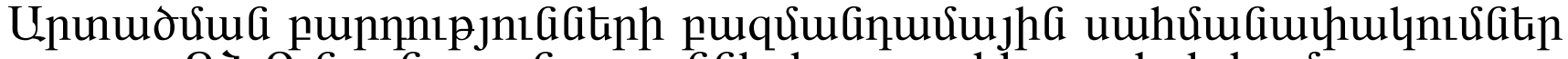

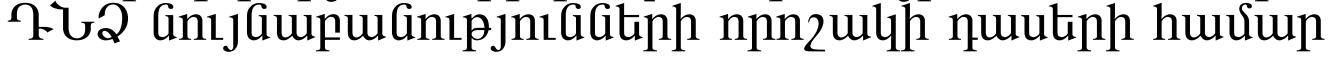

\author{
quphl Ч. Thinnnujua

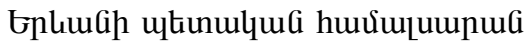 \\ e-mail: garik.petrosyan.1@gmail.com
}

\begin{abstract}
Uরưnnนnนư

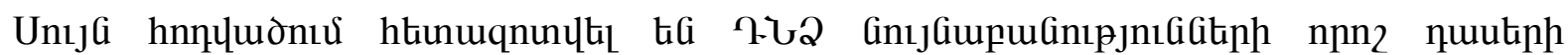

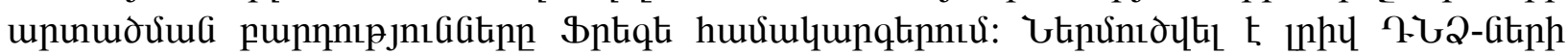

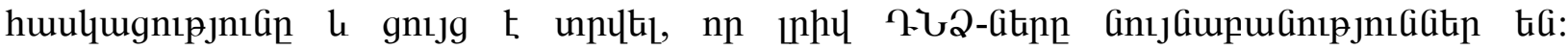

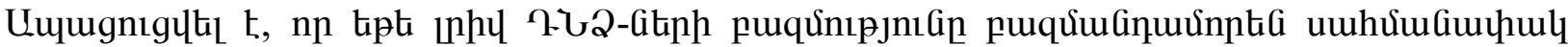

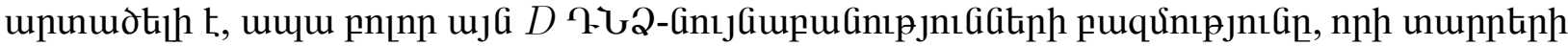

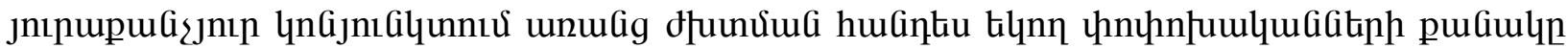

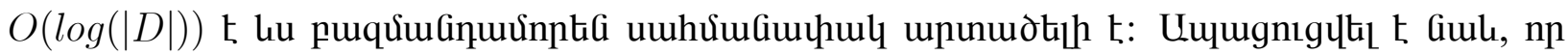

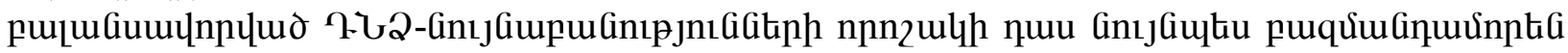
umhर्uagumuly upunudtip t:
\end{abstract}

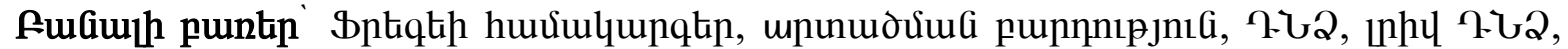

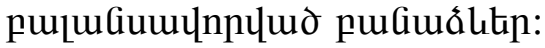

\section{Полиномиальное ограничение сложностей выводов некоторых классов $А Н Ф$-тавтологий}

\author{
Гарик В. Петросян \\ Ереванский государственный университет \\ e-mail: garik.petrosyan.1@gmail.com
}

\begin{abstract}
Аннотация
В этой статье исследованы сложности выводов в системах Фреге для некоторых классов $\lambda$ НФ-тавтологий. Введено понятие полных $\lambda$ НФ и доказано, что полные $\lambda$ НФ являются тавтологиями и, если множество полных $A$ НФ имеет полиномиально ограниченные сложности выводов, то множество всех $А$ НФтавтологий $D$ в каждом конъюнкте которых число неотрицательных переменных является $O(\log (|D|))$, также имеет полиномиально ограниченные сложности выводов. Аалее доказано, что некоторый класс сбалансированных АНФтавтологий также имеет полиномиально ограниченные сложности выводов.

Ключевые слова: системы Фреге, сложности выводов, АНФ, полные $А$ НФ, сбалансированные формулы.
\end{abstract}

